been constantly elevated, at times considerably so, since the date of the confinement. It never reached above the natural standard subsequently, and from this stage, after a lingering illness with exciting alternations of hope and fear, the patient made uniform progress, and became rapidly convalescent.

CASE 3 (M. H-, aged sixteen).-On Aug. 4th, 1887, at the General Hospital, $I$ opened in the median line a central swelling of the lower abdomen, which from the history I thought probably arose from a ruptured cyst setting up localised peritonitis. The swelling was limited in extent, smooth, pyriform in shape, with the base upwards, and occupied the hypogastric region to within an inch of the umbilicus; so central and defined in shape was the collection that previous to her admission the girl was suspected of being pregnant. Still the history was inflammatory, though there was suppression of the eatamenia. It was tympanitic all over, the resonance appearing to pass into the surrounding intestine, or to be the effect of intestine in front. On opening the swelling, exit was given to a pint and a half of fetid pus, an evident rush of foul air being extruded from the first entrance into it. The exact cause was not found, owing to the matting together of intestine around. The space was washed out with a solution of boric acid and water, and a drainage tube put in. The patient made a good recovery.

CASE 4.-The subject of this case was also a girl, aged eighteen, who had an abdominal tumour thought to be ovarian. There was a strumous history, with pain, fever, and emaciation for several months, during which time the swelling appeared. The abdomen was resonant all over, but on careful palpation a swelling could be made ont in the hypogastric and part of the left iliac regions, and reaching mearly to the level of the umbilicus. On March 22nd, 1887, I opened it, the first incision giving vent to gas distinctly. About a pint of purulent fluid was let out. The cavity was washed out as before, a glass drainage tube being put in. Suppuration continued for some months before the opening closed, but the patient eventually did well.

There were features in common in all these cases. In weach, resonance was beyond question, being verified by others; the percussion note was distinctly indicative of the presence of air rather than fluid. In each case oas was plainly extruded from the first opening made, being recognised by hearing and by odour. The deduction is obvious that in such cases confidence in diagnosis cannot be made dependent upon any individual sign, but must be derived from a combination of different methods of examination.

Birmingham.

\section{A RELIABLE CAUTERY BATTERY FOR CONSULTING-ROOM USE.}

BY ARNOLD WOAKES, M.R.C.S., L.R.C.P. LOND., SURGEON TO THE LONDOX THROAT MOSPITAL.

A FEW years ago I constructed for my father, Dr. Edward Woakes, a battery for the electric cautery that should be economical and durable in its action, as well as always ready at a moment's notice. This particular battery has now been in almost constant daily use for a period of nearly three years, and has never failed to act when required, although it has been frequently used as often as six times in one day. On several occasions the fluid has been freshened by the addition of sulphuric acid, and the loss due to evaporation made good by the addition of fresh water, but one charge of the fluid has on the average lasted for six months without renewal. This result, combined with the frequent applications I have had for the details of its construction, has induced me to place before the profession the principles upon which it is based, and the mecessary details for constructing the same.

From the accompanying engraving, it will be seen that this battery consists of a single cell, the carbons of which always remain in the fluid, while the zincs are alone depressed, when in action, to a depth varying according to the strength of the fluid.

The chief improvements over other batteries by which I have arrived at the above results are:--1. The use of one large cell in the place of several smaller cells connected together for intensity. As a matter of fact, it is quantity, and not intensity, that is required for the cautery. One bichromate cell gives an electro-motive force of 1.8 volt, and is of quite sufficient intensity to overcome the resistance offered by the cautery, provided the conducting wires are not of great lencth ; any addition in this direction is for several reasons-of which space does not admit of description-worse than useless. 2. The plan of letting the carbon plates remain always in the fluid is a point of great importance. It is adopted in smaller batteries for other purposes, but for some reason or other, when large batteries are taken into consideration, the carbons are almost in. variably made to be raised with the zincs. By allowing them to remain always in the fluid the deleterious effects of crystallisation of salts in their pores are avoided; and since

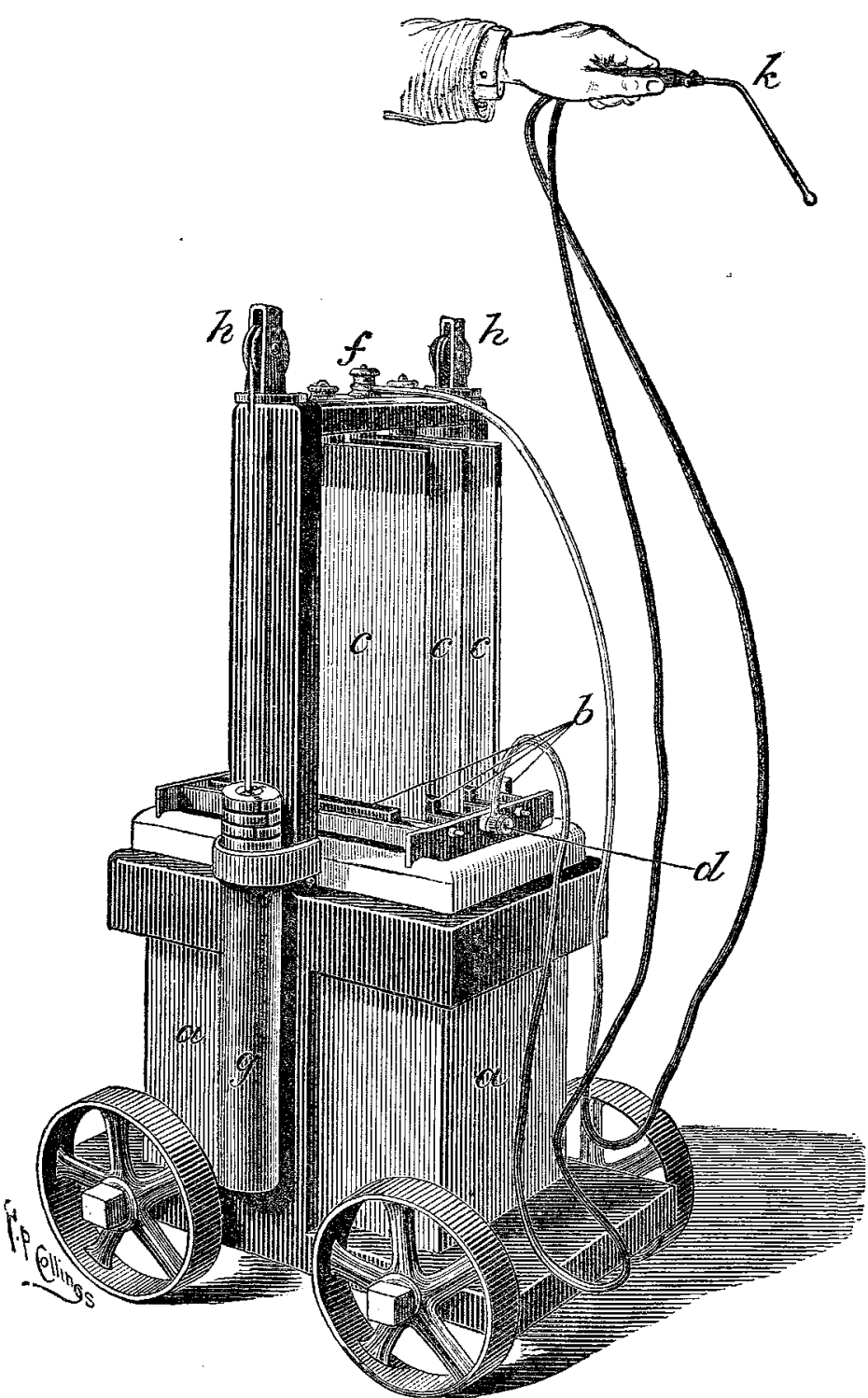

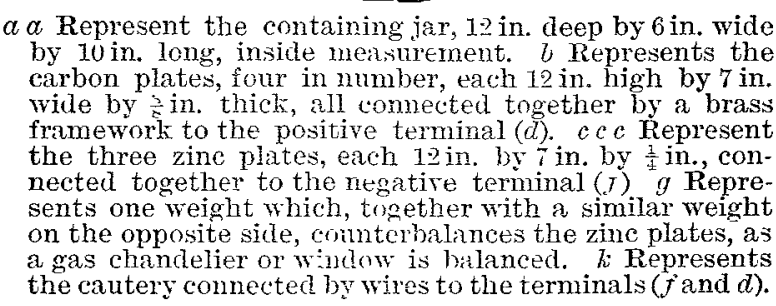

the negative and positive elements are quite distinct from one another, and not, as is usual, connected upon the same base-board, short-circuiting from the creeping of the fluid is entirely avoider: and, furthermore, the weight to be lowered and raised is diminished by the weight of the carbon plates, and there is not a single counteracting disadvantage 3. By comnterlualaneing the movable parts, the zinc plates are capable of beincr immersed with the greatest ease to any required depth, and are so easily movable that they can, if desired, be lowereit or raised by one hand of the operator, wlile with the other the cantery is still held in position. 4. In order to enable the battery to be moved about from one part of the rom to another, it is provided with wheels, but is not otherwise portable. A smaller battery upon the same plan could of course easily be con- 
structed, but only at a sacrifice of durability. Any one of the numerous bichromate solutions will answer for this battery; or a solution of chromic acid, with a little sulphuric acid, may be used instead. When the battery is to be re-charged, the exhausted fluid is syphoned off and the jar, with the carbons still in it, filled up with several re-changes of warm water. The zinc plates should at the same time be sponged over and, if necessary, re-amalgamated, but no cleaning of these plates is required in the intervals. Mr. Evans, the late porter of the London Throat Hospital, has within the past twelve months constructed several of these batteries, but without the wheels, and entirely enclosed in a wooden casing, with, I believe, entirely satisfactory results.

Harley-street, w.

\section{CASE OF}

\section{EPILEPTIFORM SEIZURE WTTH UNUSUAL} PHENOMENA.

\section{BY F. G. TOOKER, M.D. R.U.I.}

Mrs. B-, aged forty-six, married sixteen years, had a fit soon after marriage, when eight months pregnant, which cansed premature labour, with the death of the child. Six years afterwards she had another fit, the nature of which was unknown. For the next ten years she had fair health, though she was not robust. She has two children, healthy boys generally, though the eldest (aged fourteen) has suffered from infantile paralysis. The fit which is the subject of this communication took place on Jan. 19th last. When I was summoned I found the patient lying upon the sofa downstairs. She had been discovered by her husband about half an hour previously lying on the kitchen floor, where she must have lain for some ten minutes at least. She was deeply insensible, with scarcely perceptible pulse and laboured rattling respiration. The teeth were firmly clenched, with a frothy exudation from between them. The eyeballs were everted. There was no paralysis, as the arms and legs were tossed about at intervals. The bowels had acted involuntarily. The usual restorative measures were employed, and the patient's condition improved slightly. After removal to bed she lay in a heavy torpor, with much dyspnoea. Regarding her condition as epileptic, and an early improvement as likely, I left her for a short time, having ordered a stimulant treatment.

Jan. 20th.-The patient's condition is more serious. She can be roused with difficulty from the heavy collapsed state in which she lies, occasionally tossing her arms about and uttering a loud moan. Pulse almost obliterated, and very irregular. Much dyspnoea, with tracheal rattling, which masks the cardiac sounds. Lips livid, and extremities cold. The tongue appears sore, as if bitten. The somewhat ambiguous nature of the case rendered a consultation desirable, and Dr. Paget of Great Crosby and Dr. Caton of Liverpool saw the case. An exact diagnosis was difficult. Thrombosis of heart or embolism of pulmonary vessels was suggested as probable. Stimulant treatment was agreed upon, as there was great collapse. The swallowing being good, she was ordered ether and brandy by the mouth, together with some meat preparations; also ether and strophanthus tincture (three minims), every hour hypodermically, were given alternately; while nutrient enemata of beef essence were alministered every three hours by the rectum. After eight hours of this treatment the patient improved, the pulse becoming more perceptible and respiration easier. The bladder and bowels acted well and voluntarily. The urine was examined, and found to be normal. She was now ordered a mixture of tincture of strophanthus (three minims) every three hours, and ether inhalation in case of syncope.

2lst.-Pulse and respiration much improved. Consciousness still very impaired. Patient restless, but uninterested in all around. Often groans as if in pain; replies in monosyllables to questions, and expresses herself as not being in pain. Ordered four grains of carbonate of ammonia every three hours, and two ounces of champagne alternately every three hours; and to be given bromide of potassium by rectum.

22nd.-Improvement continued. Patient restless, with sleeping intervals: Heart sounds are andible : first sound indistinet; second sharp. Temperature $96^{\circ}$. More conscious. Articulates slowly, but distinctly. Complains of pain in the head. Ordered a mixture of carbonate of ammonia, bromide of potassium, and tincture of strophanthus every four hours. 23rd. - Patient more intelligent, and taking nourishment well. Pulse good.

24th.--At midnight, exactly four days from the first symptoms, there was a change; the patient then became delirious, and to-day presents considerable mental disturbance. There is great torpor, with periods of muttering delirium and restlessness. When spoken to, the answer is "Yes" or "No," indiscriminately given. At times there is. an apparent effort made to frame a correct reply to $a_{0}$ question, but the wrong word is used, or only a word-like sound is uttered ; the articulation is also very imperfect. The patient will not execute a simple movement of the hand or foot when asked; but there is no paralysis, as she often moves them freely when she requires. She will not or cannot protrude the tongue. Temperature $97 \cdot 8^{\circ}$ (highest yet attained). The bowels and bladder act voluntarily. Nourishment well taken. Ordered bromide of potassium with carbonate of ammonia every four hours.

25 th to 26 th. - The bowels and bladder now act involuntarily. There is slight anæsthesia of the right leg, and the right pupil is rather larger than the left. Intelligence more impaired; there is difficulty in attracting the patient's attention. She puts out the tongue slowly. Temperature $96^{\circ}$. The bromide of potassium was ordered to be stopped; the carbonate of ammonia to be continued, with stimulant treatment.

27th to 29th.-There is a gradual improvement. Much more consciousness. The patient promptly draws away her arm when it is pinched. There is more use of language. She can name several articles correctly after one or two attempts. She can write her own name legibly, but not so readily the names of others. On trying to read aloud, most words are blurred and indistinct. Some words she cannot say, but can write them. From this stage the patient improved steadily and generally. She became less aphasic; and now, nearly three months after her illness, she is in fair health, but still somewhat aphasic. Her memory for recent events remains much impaired since her illness.

Remarks. - Whatever diagnosis may be given to this case, the interesting features of it will, I think, remain the same. If from the history of former attacks and from the nature of this latter one we assign to it the name of epilepsy, then we have presented to us some exceedingly rare post-epileptic phenomena, as the profound collapse immediately following: and the aphasic symptoms subsequently. If we regard cardiac thrombosis as the cause, we are not without difficulty in fitting the symptoms to such a theory; thus, the former attacks and the nature of this one, as also the absence of paralysis with the aphasia, are points hard to reconcile with a thrombus and subsequent emboli in the region of Broca's convolution. Perhaps a middle course between the two views will lead nearest to the truth, and in following it one would assign an epileptic nature to the original attack, and refer the after-symptoms to the formation of a thrombus during its course. The fall upon the kitchen floor may have produced some symptoms of concussion which added to the profound collapse following the fit. The treatment affords, I think, much evidence in favour of strophanthus in desperate cardiac collapse, and also illustrates forcibly the truth that in such cases there is hope while there is life. I ans indebted to Dr. Paget for some important notes on the case.

Blundellsands.

SHEFFIELD GENERAT INFIRMARY.-The ninetyfirst report read at the annual meeting of the governors and subscribers to the institution, held on the 5th inst., states that during the past year the expenditure had exceeded the income by $£ 177$ 0s. ld., which was attributed to the falling off in the Hospital Sunday and Saturday collections, and other minor causes. The number of berls now available are 200, of which forty-four are deroted to children's cases in distinct wards. The appointments for the increase of the honorary medical staff, as named in the preceding year's annual report, were made in September last, and found to be a source of considerable importance to the efficient working of the institution. The benefit derived by a large number of patients from the "Overend Convalescent Fund" was again recorded. The report was adopted. 